\title{
Contribuiçáo do Estudo da Apatia Para a Compreensáo da Doença de Parkinson
}

\author{
Apathy Study Contribution to The Comprehension of Parkinson's Disease \\ Patricia Matos', Sofia Tavares², Edgar Martins Mesquita ${ }^{3}$
}

\section{RESUMO}

Objetivo. A apatia é reconhecida como estando presente numa variedade de perturbaçóes psiquiátricas e condiçóes neurológicas, reportando uma perturbação motivacional no comportamento orientado para objetivos. O principal objetivo do presente estudo é contribuir para a compreensão da doença de Parkinson, através da análise da relaçáo entre a apatia, aspetos demográficos (e.g., idade, sexo) e alguns aspetos clínicos (e.g., sintomas depressivos) de sujeitos com doença de Parkinson. Método. A amostra é constituída por 61 sujeitos, 30 sujeitos da comunidade (grupo náo-clínico) e 31 sujeitos com doença de Parkinson (grupo clínico), os quais foram individualmente submetidos a uma entrevista clínica e a um rastreio cognitivo. Resultados. Os resultados mostram que a apatia se apresenta como uma condição prevalente na doença de Parkinson, fortemente associada a sintomas depressivos, sobretudo no sexo feminino. Conclusóes. Os dados reforçam a necessidade de um diagnóstico eficaz da apatia na doença de Parkinson, a compreensão das suas especificidades e da sua associação com a depressão.

Unitermos. Doença de Parkinson, Apatia, Depressão.

Citaçáo. Matos P, Tavares S, Mesquita EM. Contribuição do Estudo da Apatia Para a Compreensão da Doença de Parkinson.

\begin{abstract}
Objective. Apathy is recognized as a symptom of several psychiatric and neurological disorders, reporting a disturbance in the motivational goal-directed behavior. The main objective of our research is to contribute to the understanding of Parkinson's disease through the relationship analyzes between apathy, social demographic aspects (e.g., age, sex) and some clinical aspects (e.g., depressive symptoms) of Parkinson's disease subjects. Method. The normative sample is composed of 61 subjects, 30 subjects from the community (non-clinic group) and 31 who suffer from Parkinson's disease (clinic group). Results. The results show a high prevalence of symptoms of apathy, strongly associated to symptoms of depression and prevalent on women. Conclusions. In this sense, our study reinforces the need for an effective diagnosis of apathy in Parkinson's disease, the understanding of their specific features and their association with depression.
\end{abstract}

Keywords. Parkinson Disease, Apathy, Depression.

Citation. Matos P, Tavares S, Mesquita EM. Apathy Study Contribution to The Comprehension of Parkinson's Disease.
Trabalho realizado na Universdidade de Évora, Évora, Portugal.

1.Psicóloga Clínica, Mestre em Psicologia Clínica e da Saúde, Universidade de Évora, Évora, Portugal.

2.Professora Auxiliar Convidada da Universidade de Évora, Doutora em Psicologia Clínica, Universidade de Évora, Évora, Portugal.

3.Psicólogo Clínico, Mestre em Temas de Psicologia, Núcleo de Estudantes de Estatística da Universidade do Minho, Évora, Portugal.
Endereço para correspondência: Sofia Tavares

Departamento de Psicologia, Universidade de Évora, Colégio Pedro da Fonseca, PITE Parque Industrial e Tecnológico de Évora Rua da Barba Rala, 7000 Évora, Portugal. E-mail: aireis@ualg.pt 


\section{INTRODUÇÃO}

A doença de Parkinson (DP) é uma doença neurodegenerativa progressiva, caracterizada pela perda de neurónios dopaminérgicos ao nível da substantia nigra e, em Portugal, segundo algumas estimativas, afeta mais de 20 mil pessoas ${ }^{1}$. O interesse pelos aspetos não-motores desta patologia é recente e atualmente é consensual que estes - onde se incluem, entre outros, as alteraçóes psiquiátricas - contribuem para uma grande parte da morbilidade da doença e para o agravamento da qualidade de vida dos doentes e dos seus cuidadores, podendo constituir-se como fatores de risco para a demência associada à DP e, nalguns casos, preceder os sintomas motores².

A investigação tem evidenciado a apatia enquanto uma condição presente em doenças psiquiátricas (e.g., esquizofrenia, depressão), em doenças cerebrovasculares, em condiçóes traumáticas e em doenças degenerativas (e.g., demência de Alzheimer, demência frontotemporal, coreia de Hungtinton, paralisia supranuclear progressiva $)^{3}$. A investigação de carácter longitudinal tem, aliás, evidenciado a cronicidade da apatia na demência de Alzheimer, na demência com corpos de Lewy, na esquizofrenia e na $\mathrm{DP}^{3}$.

$\mathrm{Na}$ DP a apatia constitui-se como a segunda condiçâo neuropsiquiátrica mais comum, depois da sintomatologia depressiva ${ }^{4}$, e o seu estudo foi alvo de um grande desenvolvimento na última década. A importância crescente da investigação acerca da apatia - estudada de forma pioneira por Robert Marin ${ }^{5}$-, chamou a atenção para a necessidade de uma uniformização concetual. Assim, a apatia representa uma perda primária de motivação que envolve alteraçóes na esfera afetiva, comportamental e cognitiva do comportamento orientado para objetivos ${ }^{5,6}$. No Manual de Diagnóstico e Estatística das Perturbaçóes Mentais (DSM-IV-TR ${ }^{6}$ a apatia surge em várias perturbações de personalidade secundárias a uma condição médica, embora seja referida por recurso a diferentes termos equivalentes. A apatia envolve um conjunto de sintomas, tais como: a diminuição do interesse, a diminuição da participação nas atividades da vida diária, a falta de iniciativa, a tendência para abandonar precocemente as atividades, a indiferença e a flutuação do afeto.

O estudo da apatia na DP começou a fazer-se no início da década de 90 e desde então têm-se empreendido várias investigações sobre este tópico. A etiologia da apatia na DP não está totalmente esclarecida, embora alguns estudos tenham vindo a comprovar que os indivíduos com DP exibem níveis mais elevados de apatia quando comparados com outros doentes com défices motores crónicos ${ }^{7}$.

Starkstein, Mayberg, Preziosi, Andrezejewski, Leiguarda e Robinson ${ }^{8}$ foram os primeiros a investigar a frequência de sintomas de apatia na DP. Na literatura a taxa de prevalência dos sintomas de apatia nesta condição neurológica varia entre os $27 \%$ e os $60 \%{ }^{8-11}$.

$\mathrm{Na}$ literatura surgem duas linhas de interpretação da relação entre a presença de apatia e a depressão na DP. Por um lado, alguns autores ${ }^{10,12}$ defendem o pressuposto da coexistência de depressão e apatia nesta patologia. Uma outra abordagem sustenta a apatia como um síndrome independente da presença de sintomatologia depressi$\mathrm{va}^{11}$, sugerindo que na sua origem estarão processos neuropatológicos distintos ${ }^{13}$. Por exemplo, num estudo que comparou 80 sujeitos com DP e 20 sujeitos com distonia, os autores verificaram que a apatia foi mais comum nos parkinsonianos e que esta é independente da depressão ${ }^{14}$. $\mathrm{Na}$ paralisia supranuclear progressiva e na demência fronto-temporal, existe uma prevalência de apatia superior a $80 \%{ }^{15}$ e uma baixa incidência de sintomas depressivos ${ }^{11}$. Realizar o diagnóstico diferencial entre a apatia e a depressão na DP parece assim ser fundamental, na medida em que, são condiçốes com necessidades terapêuticas específicas $^{16}$.

Têm sido várias as variáveis estudadas na sua relação com a apatia, sendo que apenas muito recentemente têm surgido dados que sustentam uma associação positiva entre a apatia e a severidade dos défices motores ${ }^{17}$, entre a apatia e os estádios evolutivos da doença de Hoehn e Yahr e, ainda, entre a apatia e a idade ${ }^{10}$. Pelo contrário, na revisão efetuada não se encontrou evidências da existência de correlaçáo entre a apatia e a dose de levedopa, as discinésias, os fenómenos de on-off e a duração da doença ${ }^{8}$. Relativamente ao sexo e à escolaridade, na revisão empreendida, náo se encontraram estudos que analisem estas variáveis na sua relação com a apatia.

Em Portugal, desconhecemos a existência de estudos especificamente dedicados ao estudo da apatia na DP. Contudo, uma equipa de investigadores tem desenvol- 
vido estudos no âmbito da validação de uma escala de apatia $^{18}$.

Em suma, a apatia está entre as condiçóes neuropsiquiátricas mais comuns na DP e, à semelhança da depressão ${ }^{19}$, a sua presença é considerada um fator de risco na demência, um fator prejudicial na funcionalidade e no domínio cognitivo, devendo por isso ser um importante foco de atenção diagnóstica e terapêutica ${ }^{9-11}$. Este trabalho tem, assim, por objetivo contribuir para a compreensão da DP através do estudo da presença de apatia e da análise da relação entre a apatia, e alguns aspetos demográficos (e.g., idade, sexo) e clínicos (e.g., sintomas depressivos) de sujeitos com DP. A pesquisa justifica-se pela necessidade de compreensão das condições psiquiátricas que podem estar presentes na DP, visto que possuem relevância para decisóes clínicas no contexto desta patologia.

\section{MÉTODO}

\section{Amostra}

Para este estudo foram recrutados 61 participantes, segundo procedimentos de amostragem por conveniência, considerando-se dois grupos: 30 sujeitos da comunidade (grupo não-clínico) e 33 sujeitos com DP (grupo clínico), tendo a sua participação obedecido a critérios de inclusão e exclusão. Foram definidos como critérios de inclusão no grupo não-clínico: (1) idade compreendida entre os 25 e os 100 anos, (2) língua materna portuguesa, (3) resultado acima do ponto de corte para a população portuguesa no Exame do Estado Mental (MMSE) e (4) ausência de história de distúrbios de desenvolvimento, perturbação psiquiátrica e défices motores, visuais ou auditivos significativos após correção. Como critério de exclusão no grupo não-clínico destaca-se (1) a presença de doença neurológica, perturbações psiquiátricas ou défice cognitivo, de acordo com os critérios clínicos do DSM-IV-TR 6 . O grupo clínico obedece aos mesmos critérios de inclusão, diferenciando-se pelo facto de ser constituído por 31 adultos com diagnóstico de DP, realizado com base nos critérios definidos pelo Banco de Cérebros da Sociedade de Parkinson do Reino Unido (UKPDDSBB). Neste sentido, foram excluídos do grupo clínico dois sujeitos pela presença de défice cognitivo.

O cumprimento de todos os critérios de inclusão teve por base uma entrevista realizada por um psicólogo, com recurso a um questionário sociodemográfico acerca de dados pessoais, história clínica e estado de saúde mental. Note-se que, apesar de não ter sido considerado critério de exclusão, o registo detalhado da toma de medicação e da história clínica foi considerada importante (nomeadamente história de cirurgias, alteraçôes tiroideia, colesterol elevado, hipertensão). A segunda fase de inclusão baseou-se no desempenho da prova de rastreio cognitivo. Os participantes do grupo clínico foram recrutados no serviço de neurologia do Hospital do Alto Ave, EPE, por reencaminhamento dos neurologistas. Neste caso, foram previamente explicados os critérios de inclusão e exclusão no estudo. Da mesma forma, o objetivo da investigaçâo foi resumidamente apresentado aos participantes, tendo sido obtido o seu consentimento informado e a autorização da Comissão de Ética do Hospital do Alto Ave, EPE (Protocolo no 18/CES).

A amostra final é composta por 61 sujeitos (25 homens e 36 mulheres) da zona norte e litoral do país (Portugal), subdivididos em dois grupos: um grupo constituído por 30 sujeitos da comunidade, com uma média de idades de 58.07 anos (DP=8.58); e outro grupo de 31 doentes com DP, com uma média de idades de 71.32 $(\mathrm{DP}=10.83)$. Quanto à escolaridade, os participantes dos grupos clínico e não-clínico têm predominantemente habilitações ao nível do Ensino Básico (77.4\% e 83.3\% respetivamente).

\section{Instrumentos e Procedimentos}

Todos os participantes foram avaliados por um psicólogo com formação em entrevista clínica, com recurso a vários instrumentos: (1) um questionário sociodemográfico e clínico, (2) o Exame de Estado Mental (MMSE) ${ }^{21}$, (3) escala de avaliação da sintomatologia depressiva (Escala de Ansiedade e Depressão Clínica - HADS ${ }^{22}$ e (4) escala de avaliação dos sintomas de apatia (Escala de Avaliação de Apatia, Versão do Profissional - EAA-P;) ${ }^{18}$. O MMSE é um teste que avalia as funçóes cognitivas de forma global, de fácil e rápida aplicação (requerendo a mesma entre 5 a 10 minutos), sendo o tempo de execução não cronometrado. É constituído por 30 questôes divididas em seis domínios cognitivos: Orientação, Retenção, Atenção e Cálculo, Evocação, Linguagem, e Habilidade Construtiva. Cada item do teste é pontuado com 
valores de 0 ou 1, variando a pontuaçáo total entre 0 e 30 valores (correspondendo este último a um melhor desempenho). A avaliação da precisão da versão portuguesa do MMSE $^{26}$ (utilizada neste estudo), realizada através da análise do valor de alpha de Cronhbach foi de 0.464, o que revela a sua adequação aos objetivos de avaliação. Os valores normativos para a população portuguesa ${ }^{21}$ são: 15 pontos (para analfabetos), 22 pontos (com uma escolaridade entre 1 a 11 anos) e 27 pontos (com uma escolaridade superior a 11 anos).

A HADS é uma escala originalmente desenvolvida por Zigmund e Snaith, que foi validada para a população portuguesa por Pais Ribeiro et al. ${ }^{22}$ e que é, na atualidade, largamente usada na investigação e prática clínica para avaliar de uma forma breve os níveis de ansiedade e de depressão. De acordo com a recomendação da Academia Americana de Neurologia, a HADS é aconselhada como ferramenta útil no estudo da depressão na doença de Parkisnon $^{23}$. Trata-se de uma escala de autorresposta, cujo preenchimento requer cerca de 10 minutos. Contém 14 itens divididos em duas subescalas, uma que mede a depressão e outra a ansiedade, cada uma composta por sete itens. Para cada um dos 14 itens da escala existem quatro possibilidades de resposta (i.e. escala de Lickert de 4 pontos) de zero a três (correspondendo o zero a inexistente e 3 a muito grave), devendo o respondente escolher aquela que melhor se adapta à forma como se tem sentido durante a última semana. Os resultados totais de cada subescala variam entre 0 e 21 , resultantes da soma dos valores dos itens de cada subescala. Os valores mais elevados em cada subescala são representativos de níveis mais elevados de ansiedade e depressão. O ponto de "corte" para a população portuguesa 11 valores em cada uma das subescalas, correspondendo os valores inferiores a ausência de ansiedade e de depressão ${ }^{22}$. A versão portuguesa apresenta dois fatores e os resultados encontrados indicam uma elevada consistência interna (alfa de Cronbach de 0.76 na subescala de ansiedade e de 0.81 na subescala de depressão).

A EAA-P24, aferida para a população portugue$\mathrm{sa}^{18}$, permite avaliar a presença de sintomas de apatia e é constituída por 15 itens, 4 dos quais são de autopreenchimento. Os itens são cotados numa escala de Lickert de 4 pontos e dividem-se em três dimensôes: apatia comportamental, apatia emocional e apatia cognitiva. Os re- sultados da escala variam entre 18 e 72 pontos, sendo os resultados mais elevados representantes de uma maior severidade de apatia. A versão portuguesa ${ }^{18}$ (por nós utilizada) apresenta um nível de fidelidade bastante satisfatório (alpha de Cronbach 0.82), com o ponto de "corte" igual ou superior a 35 pontos. Em virtude da debilidade física dos doentes de Parkinson, quando recorriam aos serviços de saúde, estes eram habitualmente acompanhados por cuidadores, que contribuíram para a avaliação realizada pelo profissional de saúde no preenchimento da EAA-P que se faça na aplicação deste instrumento ${ }^{30}$.

\section{Análise Estatística}

As análises estatísticas foram realizadas com recurso ao Statistical Package for the Social Sciences (SPSS), versão 20.0. A amostra foi caracterizada por recurso a estatísticas descritivas. A prevalência dos sintomas de apatia e de depressão foi analisada através de uma análise de frequências e do Teste do Qui-Quadrado. O pressuposto de normalidade inerente à utilização de inferência foi testado através da estatística Shapiro-Wilks. Os resultados médios da EAA-P e na subescala de depressão da HADS foram medidos através da média e respetivo desvio-padrão, e as diferenças nos resultados das mesmas escalas foram analisadas através do Teste de Mann-Whitney. A correlação entre os sintomas de apatia e depressão foi analisada com o coeficiente de correlação de Spearman, bem como a relação com as variáveis demográficas.

\section{RESULTADOS}

Os resultados do estudo da precisão das escalas dos sintomas de apatia e de depressão para a nossa amostra apresentaram índices de fiabilidade elevados, tendo os valores de alfa de Cronbach superado os limiares críticos estabelecidos na literatura ${ }^{20}$. Neste caso, os valores obtidos de 0.97 na EAA-P e de 0.89 na HADS, mostram-se adequados face aos objetivos da avaliação, sendo interessante notar que se situam acima de 0.70 .

Um dos aspetos que se procurou compreender foi a possível prevalência da apatia, em sujeitos com DP e em sujeitos ditos "normais". Verificou-se uma prevalência de apatia mais elevada nos sujeitos com doença de Parkinson (74.2\%; n=23) por comparação com o grupo não-clínico $(13.3 \% ; n=4)$, sendo esta diferença estatisticamente sig- 
nificativa $\left(\chi^{2}=22.89, \mathrm{gl}=1, \rho \leq .001\right)$. A taxa de prevalência de sintomatologia depressiva no grupo clínico foi de $41.9 \%$ ( $n=13)$, por comparação com o grupo não-clínico $(0 \% ; \mathrm{n}=0)$, sendo esta diferença estatisticamente significativa $\left(\chi^{2}=15.98, \mathrm{gl}=1, \rho \leq .001\right)$.

Depois de analisada a prevalência de apatia e de depressão, compararam-se os grupos clínico e não-clínico quanto aos valores médios obtidos na EAA-P e na subescala de depressão da HADS (Tabela 1 e 2). Uma vez que o pressuposto de normalidade não foi cumprido no grupo não-clínico (Shapiro-Wilks, $\rho \leq .0 .01$ ) recorreu-se ao teste não-paramétrico Mann-Whitney para comparar os grupos. A pontuação na EAA-P foi mais elevada no grupo clínico $(\mathrm{M}=45.06, \mathrm{DP}=12.55)$, face ao grupo não-clínico $(\mathrm{M}=26.50, \mathrm{DP}=6.95)$, sendo a diferença observada estatisticamente significativa $(\rho \leq .0 .01)$. Na análise comparativa dos valores médios obtidos na subescala de depressão da HADS, no grupo clínico e não-clínico, verificou-se a mesma tendência nos resultados. Ou seja, os sujeitos do grupo não-clínico apresentaram em média 3.45 pontos $(\mathrm{DP}=2.11)$ na subescala de depressão da HADS, enquanto os sujeitos do grupo clínico apresentaram, em média, 10.71 pontos $(\mathrm{DP}=4.63)$. Devido ao incumprimento do pressuposto da normalidade no grupo

Tabela 1

Valores médios e desvio-padrão dos resultados na Escala de Avaliação de Apatia - Versão do Profissional nos grupos clinico e não-clínico

\begin{tabular}{cccccc}
\hline Grupo & $\mathrm{n}$ & Média & $\begin{array}{l}\text { Desvio } \\
\text { Padrão }\end{array}$ & & \multicolumn{2}{c}{ Diferenças } & \\
\cline { 5 - 6 } & & & & & \\
& & & & \\
Grupo & & & $\rho$ \\
náo-clínico & 30 & 26.50 & 6.95 & & \\
$\begin{array}{c}\text { Grupo } \\
\text { clínico }\end{array}$ & 31 & 45.06 & 12.55 & & \\
\hline
\end{tabular}

${ }^{a}$ Teste Mann-Whitney

${ }^{*} \rho \leq .001$

Tabela 2

Valores médios e desvio-padrão dos resultados na Escala de Depressão Clínica (HADS) nos grupos clínico e não-clínico

\begin{tabular}{|c|c|c|c|c|c|}
\hline \multirow{2}{*}{ HADS } & \multirow{2}{*}{$\mathrm{N}$} & \multirow{2}{*}{ Média } & \multirow{2}{*}{$\begin{array}{l}\text { Desvio } \\
\text { padrấo }\end{array}$} & \multicolumn{2}{|c|}{ Diferenças } \\
\hline & & & & $\mathrm{M}-\mathrm{W}^{\mathrm{a}}$ & $\rho$ \\
\hline $\begin{array}{c}\text { Grupo } \\
\text { não-clínico }\end{array}$ & 30 & 3.45 & 2.11 & \multirow{2}{*}{68.00} & \multirow{2}{*}{$.001^{*}$} \\
\hline $\begin{array}{l}\text { Grupo } \\
\text { clinico }\end{array}$ & 31 & 10.71 & 4.63 & & \\
\hline
\end{tabular}

${ }^{\text {a }}$ Teste Mann-Whitney

${ }^{*} \mathrm{p} \leq .001$ não-clínico foi utilizado o teste de Mann-Whitney, sendo a diferença observada uma vez mais estatisticamente significativa $(\rho \leq .001)$.

Procedeu-se, de seguida, a um conjunto de operaçôes e de testes estatísticos para averiguar a natureza da associação entre a apatia e a depressão, tendo em consideração os sujeitos com apatia do grupo de doentes de Parkinson ( $\mathrm{n}=23)$. A taxa de prevalência de sintomatologia depressiva foi de $56.52 \%$ ( $n=13$ ), sendo que $43.48 \%$ $(\mathrm{n}=10)$ dos sujeitos com apatia não apresentaram sintomas de depressão. Nenhum dos sujeitos do grupo clínico sem apatia apresentou sintomatologia depressiva $(n=8)$.

Depois de analisada a frequência dos sintomas de apatia e depressão, testou-se o grau de associação entre esta sintomatologia. Para tal, calcularam-se os coeficientes de correlação de Spearman (Shapiro-Wilks, $\rho \leq .001$ ), entre o resultado na EAA-P e na subescala de depressão da HADS, em cada um dos subgrupos. Pela correlação dos resultados obtidos nas escalas, no grupo de sujeitos com doença de Parkinson, os resultados na EAA-P encontram-se positivamente associados e de forma elevada aos resultados na subescala de depressão da HADS $(\mathrm{r}=.80, \rho \leq .001)$. Assim, de um modo global, os sujeitos que apresentaram sintomas de apatia evidenciaram também sintomatologia depressiva, sendo que esta sintomatologia tende a ser mais elevada quanto mais elevados forem os sintomas de apatia. Pelo contrário, os resultados mostraram que, no subgrupo de participantes da comunidade com apatia $(n=4)$, os resultados na EAA-P não se encontraram positivamente associados aos resultados na subescala de depressão da HADS.

Outro resultado que se evidencia é uma idade média mais elevada nos sujeitos com sintomas de apatia. Neste caso, os sujeitos sem apatia do grupo clínico $(n=8)$ apresentaram em média 64.00 anos de idade ( $\mathrm{DP}=14.70)$, enquanto os sujeitos deste grupo com apatia $(n=23)$ apresentaram, em média, 73.87 anos de idade $(\mathrm{DP}=8.05)$. No grupo náo-clínico, os sujeitos sem apatia $(n=26)$ apresentaram, em média, 57.96 anos de idade $(\mathrm{DP}=8.63)$, enquanto os sujeitos com apatia $(\mathrm{n}=4)$ apresentam, em média, 58.75 anos de idade (DP=9.54). As diferenças observadas nos grupos clínico e no grupo não-clínico em relação à idade média dos participantes foram medidas com o recurso ao Teste Mann-Whitney 
(Shapiro-Wilks, $\rho \leq .01 \mathrm{em}$ ambos os grupos) e os resultados foram marginalmente significativos para o grupo clínico ( $\rho \leq .10$; cf. sugestão de Tabachnick $\&$ Fidell $^{26}$ para amostras reduzidas).

Numa outra análise, testou-se o grau de associação entre a pontuação na escala de apatia e a idade na amostra, a qual não se revelou estatisticamente significativa quer no grupo clínico quer no grupo não-clínico. De forma semelhante, não se verificou uma relação estatisticamente significativa na análise da associação entre a idade e a sintomatologia depressiva.

Outro dos aspetos que se procurou compreender foi a possível prevalência de sintomas de apatia e de depressão em função do sexo. Assim, no que respeita à presença de sintomas de apatia no grupo clínico, verificou-se uma prevalência de apatia mais elevada nas mulheres (85\%; $\mathrm{n}=17)$ do que nos homens $(54.5 \% ; \mathrm{n}=7)$, sendo a diferença marginalmente significativa $\left(\chi^{2}=3.44, \mathrm{gl}=1\right.$, $\rho \leq .10)$. À semelhança destes resultados, e no que respeita à presença de sintomas de depressão no grupo clínico, verificou-se também que as mulheres apresentam uma prevalência mais elevada $(55 \% ; \mathrm{n}=11)$ do que os homens $(18.2 \%$; $n=2)$, sendo a diferença estatisticamente significativa $\left(\chi^{2}=3.95, \mathrm{gl}=1, \rho \leq .05\right)$. No grupo não-clínico não se verificaram diferenças estatisticamente significativas na presença de sintomas de apatia e de depressão em função do sexo.

\section{DISCUSSÃO}

Neste estudo procura-se investigar a presença de apatia e analisar a eventual relação entre esta e alguns aspetos demográfico e clínicos de sujeitos com DP. Os resultados sugerem que a apatia é mais comum em sujeitos com DP por comparação com os sujeitos da população geral e que se manifesta com mais intensidade nesta doença. A prevalência da apatia na DP - que no presente estudo atinge valores ainda mais elevados do que os encontrados em estudos anteriores -, é concordante com os dados de outras investigações $^{8-10}$. Os estudos revelam inconsistências nas taxas de apatia apresentadas para esta populaçáo, que podem ser devidas a fatores como: a complexidade e a variedade de critérios de diagnóstico e de definiçóes usadas para o síndrome de apatia, o recurso a diferentes técnicas de avaliação e às características da própria amostra.
Globalmente, os dados deste estudo sugerem que perante o diagnóstico de uma doença neurodegenerativa como a DP é elevada a probabilidade de simultaneamente se encontrarem presentes sintomas de apatia. Estes resultados devem ser interpretados com reserva na medida em que alguns dos sujeitos com DP da amostra se encontravam em internamento, sendo que esta circunstância, associada à eventual toma de medicação, pode ter influenciado os resultados obtidos na EAA-P. Para além disso, nalguns casos a cotação da EAA-P baseou-se apenas na entrevista ao sujeito com DP, sabendo que a complementar informação proveniente de outras fontes é fundamental.

A presença de sintomatologia depressiva nos doentes de Parkinson da nossa amostra apresenta o mesmo padrão de outros estudos ${ }^{27}$, com os resultados a sugerir que na DP os sintomas de depressão são uma condição prevalente. A pertinência da análise deste fenómeno prende-se com a consideração de que os sintomas depressivos podem preceder até o aparecimento das alteraçóes motoras que levam ao diagnóstico da doença ${ }^{28}$. Os nossos dados vão também de encontro à visão presente na literatura empírica de que a sintomatologia depressiva é uma condição mais comum nas mulheres do que nos homens.

A presença de sintomas de apatia na DP é conhecida, mas a sua relação com a sintomatologia depressiva tem gerado alguma controvérsia, com alguns estudos a sugerir a ocorrência simultânea de apatia e depressão na DP", enquanto outros não confirmam uma associação estatisticamente significativa ${ }^{11}$. Neste estudo, verificou-se que, para além da propensão geral verificada na totalidade da amostra, nos doentes de Parkinson existe uma associação mais forte entre sintomas de apatia e de depressão. A presença conjunta de apatia e de depressão nesta patologia é explicada pela base comum de alterações neuropatológicas e neuroquímicas associadas à doença, ou pelos instrumentos de medida utilizados que contêm itens transversais às duas condições - nomeadamente, itens relacionados com sintomas de fadiga, perda de interesse, anedonia e lentificação psicomotora ${ }^{10,12}$. Uma das hipóteses explicativas da comorbilidade encontrada no presente estudo poderá residir no facto do levantamento de sintomas depressivos ser realizado na ausência do critério "humor deprimido" e da escala de avaliação usada (i.e., HADS) incluir um item relativo à perda de interesse. 
A influência das variáveis sociodemográficas não tem sido consistentemente considerada na literatura. Neste estudo, a idade não se encontra correlacionada de forma positiva e estatisticamente significativa com os sintomas de apatia, embora os doentes de Parkinson com apatia sejam mais velhos. Este resultado não é congruente com o único estudo encontrado ${ }^{10}$, que apresenta uma relação entre a idade e a apatia. No entanto, os dados do nosso estudo não acompanham os resultados da literatura que apresentam uma associação entre a idade e a sintomatologia depressiva.

Este estudo apresenta como limitaçóes: (1) a opção de utilização da Escala de Avaliação de Apatia, que apresenta alguns itens de autopreenchimento; (2) o facto do diagnóstico de depressão na HADS ser efetuado na ausência de humor deprimido, o que poderá sugerir que a depressão leve na DP pode remeter para um quadro de apatia, mais do que uma real perturbação emocional; (3) o processo de amostragem por conveniência que restringe o alcance dos resultados obtidos. Para além destas, (4) a toma de medicação não foi controlada, podendo esta variável interferir com os nossos resultados, na medida em que a evidência empírica ${ }^{30}$ sugere que, por um lado, os défices motivacionais podem ser potenciados pela prescrição medicamentosa e que, por outro, a medicação antidepressiva poderá ter um efeito positivo nos sintomas de apatia ${ }^{31}$. Por último, (5) uma das limitaçóes do presente estudo diz respeito à heterogeneidade da amostra, no que respeita a alguns aspetos clínicos (e.g., idade de início da DP, gravidade clínica da DP através do estadiamento de Hoehn e Yahr). O facto de não ser registado o período de flutuaçóes motoras (i.e., estádio on-off), tendo em conta que são comuns em fases mais avançadas da doença, merece também uma reflexão em termos de limitações. Durante estas fases os pacientes podem percecionar os seus comportamentos e sentimentos de forma diferente, o que consequentemente pode influenciar as suas respostas ${ }^{32}$. De referir ainda, que é importante estudar este fenómeno no período off da doença, uma vez que, por exemplo, foram registados nesta fase da doença sintomas de depressão e de ansiedade ${ }^{33}$.

\section{CONCLUSÕES}

Este estudo explora a incidência de apatia e a sua relação com outras alteraçóes clínicas e alguns aspetos sociodemográficos na DP. O tema acompanha a atual tendência da comunidade científica ao debruçar-se sobre os aspetos não-motores da DP e parece-nos de grande pertinência clínica: a identificação de sintomas de apatia pode ajudar a estabelecer o diagnóstico e também pode ser um elemento preditor da demência associada à DP. É também importante a identificação desta sintomatologia para que se possa implementar um acompanhamento adequado. No futuro, esperamos que se desenvolvam estudos com grupos de comparação constituídos por sujeitos com doenças motoras crónicas e progressivas semelhantes à DP e em amostras mais homogéneas (estágio de evolução da doença e período de flutuaçóes motoras).

\section{AGRADECIMENTOS}

À diretora do Serviço de Neurologia do Hospital do Alto Ave, EPE, Dr. ${ }^{a}$ Maria de Lurdes Rodrigues. À Dra. Maria José Jordão, do Serviço de Neurologia do Hospital do Alto Ave, EPE.

\section{REFERÊNCIAS}

1.Programa nacional para a saúde das pessoas idosas (endereço na Internet). Ministério da educação: Direção Geral de Saúde (atualizado 7/2004; acessado em 07/2011). Disponível em: http://www.portaldasaude.pt/NR/ rdonlyres/1C6DFF0E-9E74-4DED-94A9-F7EA0B3760AA/0/i006346.pdf

2.Barone P. Neurotransmission in Parkinson's disease: beyond dopamine. Eur J Neurol 2010;17:364-76.

http://dx.doi.org/10.1111/j.1468-1331.2009.02900.x

3.Chase, TN. Apathy in neuropsychiatric disease: Diagnosis, pathophysiology and treatment. Neurotox Res 2011;19:266-78.

http://dx.doi.org/10.1007/s12640-010-9196-9

4.Chagas MH, Crippa JA, Tumas V. Antidepressivos no Tratamento de Depressão na Doença de Parkinson: Quais as Evidências? Rev Neurocienc 2011;19:570-572.

5.Marin RS. Differential diagnosis and classification of apathy. Am J Psychiatry 1990;147:22-30.

6.American Psychiatric Association. DSM-IV-TR: Manual de diagnóstico e estatística das perturbaçóes mentais (4a ed.). Portugal: Climepsi Editores, 1994, 800 p.

7.Zgaljardic DJ, Foldi NS, Borod JC. Cognitive and behavioral dysfunction in Parkinson's disease: neurochemical and clinicopathological contributions. J Neural Transm 2004;111:1287-301.

http://dx.doi.org/10.1007/s00702-004-0178-z

8.Starkstein SE, Mayberg H S, Preziosi T J, Andrezejewski P, Leiguarda R, Robinson RG. Reliability, validity, and clinical correlates of apathy in Parkinson's disease. J Neuropsychiatry Clin Neurosis 1992;4:134-39. 
9.Dujardin K, Sockeel P, Delliaux M, Destée A, Defebvre L. Apathy may herald decline and dementia in Parkinson's disease. Mov Disord 2009;24:2391-397. 10. Orugu M, Tachibana H, Toda K, Okuda B, Oka, N. Apathy and depression in Parkinson's disease. J Geriat Psychiatry and Neurol 2010;23;35-41. http://dx.doi.org/10.1177/0891988709351834

11.Pluck GC, Brown RG. Apathy in Parkinson's disease. J Neurol Neurosurg Psychiatry 2002;73:636-42.

http://dx.doi.org/10.1136/jnnp.73.6.636

12.Pedersen KF, Larsen JP, Alves G, Aarsland D. Prevalence and clinical correlates of apathy in Parkinson's disease: A community-based study. Parkinsonism Relat Disord 2009;15:295-99.

http://dx.doi.org/10.1016/j.parkreldis.2008.07.006

13.Zgaljardic D J, Borod JC, Foldi NS, Mattis P. A review of the cognitive and behavioral sequelae of Parkinson's disease: relationship to frontostriatal circuitry. Cogm Behavl Neurol 2003;16:193-210.

http://dx.doi.org/10.1097/00146965-200312000-00001

14.Kirsch-Darrow L, Fernandez HH, Marsiske M, Okun M, Bowers D. Dissociating apathy and depression in Parkinson disease. Neurology 2006;67:33-38. http://dx.doi.org/10.1212/01.wnl.0000230572.07791.22

15.Emre M, Aarsland D, Brown R, Burn DJ, Duyckaerts C, Mizuno Y, et al. Clinical diagnostic criteria for dementia associated with Parkinson's disease. Mov Disord 2007;22;1689-707.

http://dx.doi.org/10.1002/mds.21507

16.Goetz CG. New developments in depression, anxiety, compulsiveness and hallucinations in Parkinson's disease. Mov Disord 2010;25:104-09.

http://dx.doi.org/10.1002/mds.22636

17.Pedersen KF, Larsen JP, Alves G, Aarsland D. Prevalence and clinical correlates of apathy in Parkinson's disease: A community-based study. Parkinsonism Relat Disord 2009;15:295-99.

http://dx.doi.org/10.1016/j.parkreldis.2008.07.006

18.Caeiro L, Silva T, Ferro FM, Pais-Ribeiro JP, Figueira L. Metric properties of the portuguese version of apathy evaluation scale. Psic, Saúde \& Doenças 2012;13:266-82.

19.Silva FS, Pabis JV, Alencar AG, Silva KB, Navarro-Peternella FM. Evolução da doença de Parkinson e comprometimento da qualidade de vida. Rev Neurocienc 2010;18:463-468.

20.Nunnaly J. Psychometric theory. New York: McGraw-Hill, 1978, 701p.

21.Guerreiro M, Silva AP, Botelho MA., Leitão O, Castro-Caldas A, Garcia C. Adaptaçáo à população portuguesa e tradução do Mini Mental State Examination (MMSE). Rev Port Neurol 1978;1:9-14.

22.Pais-Ribeiro J, Silva I, Ferreira T, Martins A, Meneses R, Baltar M. Vali- dation study of a Portuguese version of the Hospital Anxiety and Depression Scale. Psychol Health Med 2007;12:225-37.

http://dx.doi.org/10.1080/13548500500524088

23.Miyasaki JM, Shannon K, Voon V, Ravina B, Kleiner-Fisman G, Anderson K, et al. Quality Standards Subcommittee of the American Academy of Neurology. Practice Parameter: evaluation and treatment of depression, psychosis, and dementia in Parkinson disease (an evidence based review): report of the Quality Standards Subcommittee of the American Academy of Neurology. Neurology 2006;66(7):996-1002.

http://dx.doi.org/10.1212/01.wnl.0000215428.46057.3d

24.Marin RS, Biedrzycki RC, Firinciogullari S.. Reliability and validity of the apathy evaluation scale. Psychiatry Research 1991;38:143-162.

http://dx.doi.org/10.1016/0165-1781(91)90040-V

25.Marin RS. Guidelines for apathy evaluation scale (endereço na Internet). Australia: Dementia Collaborative Research Centres - DCRC (atualizado em 2007; acessado em 2011). Disponivel em: http://www.dementia-assessment. com.au/symptoms/AES_Guidelines.pdf

26.Tabachnick BG, Fidel LS. Using multivariate statistics. New York: Harper Collins, 1996, 983p.

27.Cummings JL. Depression and Parkinson's disease: a review. Am J Psychiatry 1992;149:443-54.

28.Ishihara L, Brayne C. A systematic review of depression and mental illness preceding Parkinson's disease. Acta Neurol Scand 2006;113:211-20.

http://dx.doi.org/10.1111/j.1600-0404.2006.00579.x

29.Levy ML, Cummings JL, Fairbanks LA, Masterman D, Miller BL, Craig AH, et al. Apathy is not depression. J Neuropsychiatry Clin Neurosci 1998;10:314-19.

30.Lee SI, Keltner NL. Antidepressant apathy syndrome. Perspect Psychiatr Care 2005;1:188-92.

http://dx.doi.org/10.1111/j.1744-6163.2005.00033.x

31.Benoit M, Andrieu S, Lechowski L, Gillette-Guyonnet S, Robert PH, Vellas B. Apathy and depression in Alzheimer's disease are associated with functional deficit and psychotropic prescription. Int J Geriatr Psychiatry 2008;23:409-14. http://dx.doi.org/10.1002/gps. 1895

32.Leentjens AF, Dujardin K, Marsch L, Martinez-Martin P, Richard, IH, Starkstein SE, et al. Apathy and anhedonia rating scales in Parkinson's disease: critique and recommendations. Mov Disord 2008;23:2004-014.

http://dx.doi.org/10.1002/mds.22229

33.Aarsland D, Marsh L, Schrag A. Neuropsychiatric symptoms in Parkinson's disease. Mov Disord 2009; 24:2175-186.

http://dx.doi.org/10.1002/mds.22589 